Journal of Environmental
ASSN: $2525-815 \mathrm{X}$

\title{
Índices biofísicos e o saldo de radiação à superfície via sensoriamento remoto no Semiárido Pernambucano
}

\section{Biophysical indexes and the net radiation at surface by remote sensing in the Pernambucano semiarid}

Landson Carlos da Silva ${ }^{\mathrm{a}}$, Jhon Lennon Bezerra da Silva ${ }^{\mathrm{b}}$, Geber Barbosa de Albuquerque Moura ${ }^{\mathrm{a}}$, Douglas Alberto de Oliveira Silva ${ }^{\mathrm{b}}$, Pabrício Marcos Oliveira Lopes ${ }^{\mathrm{a}}$, Cristina Rodrigues Nascimento $^{\mathrm{a}}$, Marcos Vinícios da Silva ${ }^{\mathrm{b}}$, Pedro Henrique Dias Batista ${ }^{\mathrm{b}}$

${ }^{a}$ Universidade Federal Rural de Pernambuco-UFRPE, Departamento de Agronomia. Av. D. Manoel de Medeiros, SN, Dois Irmãos, Recife, Pernambuco, Brasil. CEP: 52171-900. E-mail: geber@depa.ufrpe.br, pabriciope@gmail.com, crisrodnas@gmail.com, landson.14@gmail.com (Autor para correspondência).

b UFRPE, Departamento de Engenharia Agrícola. E-mail: ihonlennoigt@hotmail.com, douglasalbertosilva@hotmail.com, marcolino_114@hotmail.com, giga_pedro@ hotmail.com.

\begin{abstract}
A R T I C L E I N F O
Recebido 05 Nov 2020

Aceito 14 Jan 2021

Publicado 28 Jan 2021

A B S T R A C T

The Pernambuco semiarid has been suffering from various processes of environmental degradation due to the advancement of agricultural and livestock activities and the severe drought that afflicts the Brazilian semiarid and the Caatinga biome. The use of geoprocessing in remote sensing techniques stands out for the high applicability in environmental monitoring in the estimation of biophysical parameters of the terrestrial surface's radiation. Thus, the objective was to monitor and investigate environmental changes through spatiotemporal modeling via remote sensing using satellite images, referring to the study area, the municipality of Arcoverde, located in the Pernambuco backwoods, Brazilian semiarid. The study was developed through six images of the Landsat satellite between 2008 and 2017. The images and thematic maps of the surface were processed using the SEBAL (Surface Energy Balance Algorithms for Land) algorithm, which determined biophysical parameters such as albedo and surface temperature, and net radiation. The results showed that the highest albedo values were verified in climatically dry years $(11 / 14 / 2015$ and $09 / 29 / 2016)$, with mean values of 0.22 and 0.20 , respectively. The day $11 / 14 / 2015$ also presented the highest average value of the temperature, of $47^{\circ} \mathrm{C}$, when the balance of radiation was smaller. On 11/13/2009 (rainy year) presented the highest average value of the balance, $800 \mathrm{~W} \mathrm{~m}^{-2}$, with more available energy to be allocated by energy balance processes. The increase of the albedo and surface temperature and decrease of the radiation balance highlighted changes in the semiarid region's land uses.
\end{abstract}

Keywords: Surface albedo, surface temperature, surface radiative balance.

\section{R E S U M O}

O semiárido pernambucano vem sofrendo com diversos processos de degradação ambiental, devido ao avanço das atividades agrícolas, além da atenuante seca que assola o semiárido brasileiro e o bioma Caatinga. O uso do geoprocessamento diante das técnicas de sensoriamento remoto se destaca pela alta aplicabilidade no monitoramento ambiental, na estimativa de parâmetros biofísicos do balanço de radiação da superfície terrestre. Assim, objetivou-se monitorar e investigar as mudanças ambientais através da modelagem espaço-temporal via sensoriamento remoto com o uso de imagens de satélite, referentes à área de estudo, o município de Arcoverde, localizado no sertão de Pernambuco, semiárido brasileiro. O estudo foi desenvolvido por meio de seis imagens do satélite Landsat, entre o período de 2008 e 2017. Através das imagens foram processados mapas temáticos da superfície, 
mediante o algoritmo SEBAL (Surface Energy Balance Algorithms for Land), que determinou parâmetros biofísicos como albedo e temperatura da superfície, e saldo de radiação. Os resultados destacaram que os maiores valores do albedo foram verificados em anos climaticamente secos (14/11/2015 e 29/09/2016), com valores médios de 0,22 e 0,20, respectivamente. O dia 14/11/2015 também apresentou o maior valor médio da temperatura, de $47^{\circ} \mathrm{C}$, épocas que o saldo de radiação foi menor. O dia 13/11/2009 (ano chuvoso) apresentou o maior valor médio do saldo, de $800 \mathrm{~W} \mathrm{~m}^{-2}$, tendo maior energia disponível a ser repartida pelos processos do balanço de energia. $\mathrm{O}$ aumento do albedo e temperatura da superfície e diminuição do saldo de radiação destacaram mudanças nos usos do solo da região semiárida.

Palavras-Chave: Albedo da superfície, temperatura da superfície, balanço radioativo à superfície.

\section{Introdução}

O semiárido brasileiro vem sofrendo mudanças na sua paisagem ao longo do tempo. Nos últimos anos aumentaram os níveis de degradação ambiental e desertificação em diversas regiões do semiárido, tal qual possui uma vegetação característica do bioma Caatinga, que por sinal tem sido constantemente modificado. Todavia, os longos períodos de seca severa, desmatamento, queimadas e o avanço das atividades agrícolas instaladas e manejadas de forma inadequada tem favorecido a desequilíbrios ambientais constantes no semiárido (Gutiérrez et al., 2014; Marengo et al., 2016; Marengo et al., 2018; Mariano et al., 2018; Silva et al., 2019).

Nesse sentido, visando o monitoramento ambiental destas regiões, especialmente do semiárido pernambucano, na qual se localiza a área de estudo, torna-se essencial o uso de geotecnologias eficazes na gestão e planejamento na tomada de decisões, suprindo a ausência de dados meteorológicos e informações ambientais, facilitando assim o monitoramento da Caatinga, bem como, destacar a dinâmica da cobertura vegetal natural de Caatinga da região semiárida. $\mathrm{O}$ conhecimento e a compreensão das alterações do clima local e, ainda, como funciona as componentes do balanço de energia destas regiões são de extrema importância, especialmente nos estudos de mudanças climáticas, como por exemplo, nas pesquisas do balanço de energia à superfície terrestre, que destacam parâmetros biofísicos como o albedo e temperatura da superfície, excelentes indicadores de áreas degradadas, assim como, também destaca-se o saldo de radiação à superfície, diretamente influenciado pelo albedo e temperatura (Lins et al., 2017; Silva et al., 2017; Silva et al., 2019).

Tendo como base características inerentes à superfície, quanto a sua capacidade de absorver e refletir a radiação solar, pode-se monitorar áreas de risco e que estão sendo degradadas, levando-se em consideração que regiões não vegetadas refletem uma maior quantidade de radiação em relação as áreas com vegetação densa arbórea, tornando relativamente simplificada uma correlação da vegetação com índices biofísicos como albedo e temperatura da superfície (Arraes et al., 2012).
O geoprocessamento assegura diante do uso e aplicação de geotecnologias um monitoramento ambiental prático, eficiente e adequado, principalmente no semiárido brasileiro, sendo as técnicas de sensoriamento remoto conjuntas as imagens de satélite ferramentas de destaque na investigação de mudanças ambientais a partir de uma modelagem espaço-temporal em larga escala, tornando-se possível a detecção de parâmetros biofísicos, averiguação dos danos causados aos recursos hídricos e naturais, a exemplo do bioma Caatinga, seja pelas ações antrópicas e/ou eventos de seca (Giongo \& Vettorazzi, 2014; Ballén et al., 2016; Macedo et al., 2017; Silva et al., 2017; Silva et al., 2019).

O sensoriamento remoto possui grande vantagem no suporte e na obtenção de resultados confiáveis de fenômenos meteorológicos e ambientais, realizando um papel fundamental para compreensão precisa da dinâmica do ambiente natural e/ou degradado, especialmente quando se associa com informações meteorológicas e morfológicas da área de estudo (Meneses, 2012; Leite et al., 2017). Deste modo, diversos pesquisadores se utilizam deste tipo de aplicação, recomendando manejos adequados a partir dos estudos de análise e detecção de mudanças da cobertura vegetal, favorecendo um planejamento de gestão ambiental e orientação de medidas de mitigação e exploração dos recursos hídricos e naturais de forma sustentável no semiárido (Silva et al., 2017; Silva et al., 2019).

No sensoriamento remoto, a modelagem espaço-temporal das componentes do balanço de radiação e energia se dão pelo uso de algoritmos, essenciais na extração de informações de imagens de satélite em escala regional e global, que se configura como uma alternativa de baixo custo operacional (Bastiaanssen, 2000; Allen et al., 2002; Silva et al., 2017; Silva et al., 2019).

O algoritmo SEBAL (Surface Energy Balance Algorithm for Land), por exemplo, foi desenvolvido especificamente para estimar parâmetros biofísicos do balanço de energia à superfície e a evapotranspiração por meio de relações empíricas e parametrizações físicas (Bastiaanssen et al., 1998; Bastiaanssen, 2000). 
O SEBAL é validado em estudos de grande impacto no mundo, inclusive nas regiões semiáridas do Brasil, nos estudos sobre o monitoramento de superfícies e dos diferentes usos e ocupações do solo através do desenvolvimento de mapas temáticos da superfície terrestre (Bezerra et al., 2014; Silva et al., 2016; Silva et al., 2017; Silva et al., 2019).

Diante da necessidade de monitorar fatores como a degradação e desertificação em larga escala, além de destacar as principais mudanças do ambiente natural de Caatinga perante o clima local da região semiárida, objetivou-se monitorar e investigar as mudanças ambientais a partir do geoprocessamento com uso de técnicas de sensoriamento remoto (SEBAL) mediante imagens de satélite, por meio da modelagem quantitativa e espaço-temporal de parâmetros biofísicos do balanço de energia na interface solo-atmosfera em região do semiárido pernambucano.

\section{Material e métodos}

\section{Área de localização do estudo}

O município de Arcoverde foi selecionado para o presente estudo, estando localizado entre os paralelos de $08^{\circ} 16^{\prime} 35,97^{\prime}$ ' S (UTM: $9060577 \mathrm{~m}$ ) e $08^{\circ} 31$ ' 19,18' S (UTM: $9083079 \mathrm{~m}$ ), e entre os meridianos de $36^{\circ} 53$ ' 21,11' ' W (UTM: $710016 \mathrm{~m}$ ) e $37^{\circ} 08^{\prime}$ 15,36' W (UTM: $740019 \mathrm{~m}$ ), com uma altitude variando entre 650 e $1000 \mathrm{~m}$, a oeste do meridiano de Greenwich. Arcoverde está inserido, mais especificamente, na Mesorregião do Sertão Pernambucano e na Microrregião do Sertão do Moxotó, no semiárido do estado de Pernambuco, Região Nordeste do Brasil. O município abrange uma área absoluta de $323,369 \mathrm{~km}^{2}$, possuindo vegetação característica de áreas de agreste e sertão, formada por florestas subcaducifólias e caducifólias (Beltrão et al., 2005; IBGE, 2019). A classificação climática da região segundo Köppen, é do tipo BSh - semiárido quente, com temperaturas médias anuais variando entre $12^{\circ} \mathrm{C}$ e $25^{\circ} \mathrm{C}$, verão seco e estação chuvosa compreendida entre os meses de janeiro e setembro, podendo estender-se até outubro, com precipitação pluviométrica média anual de $652,78 \mathrm{~mm}$ conforme registros de dados climatológicos do Instituto Nacional de Meteorologia (INMET) dos últimos 31 anos (1987-2017) disponibilizados pela Agencia Pernambucana de Águas e Clima (APAC) (Alvares et al., 2013; Lins et al., 2017).

A Figura 1 apresenta o mapa espacial da área de estudo, município de Arcoverde-PE, a partir de uma imagem do satélite Landsat, na composição RGB, e resolução espacial de $30 \mathrm{~m}$. Neste mapa destaca-se a localização da cidade e da estação meteorológica. A imagem foi georreferenciada no Sistema de Projeção Cartográfica UTM (Universal Transversa de Mercator) DATUM: WGS1984 - Zona 24 L.


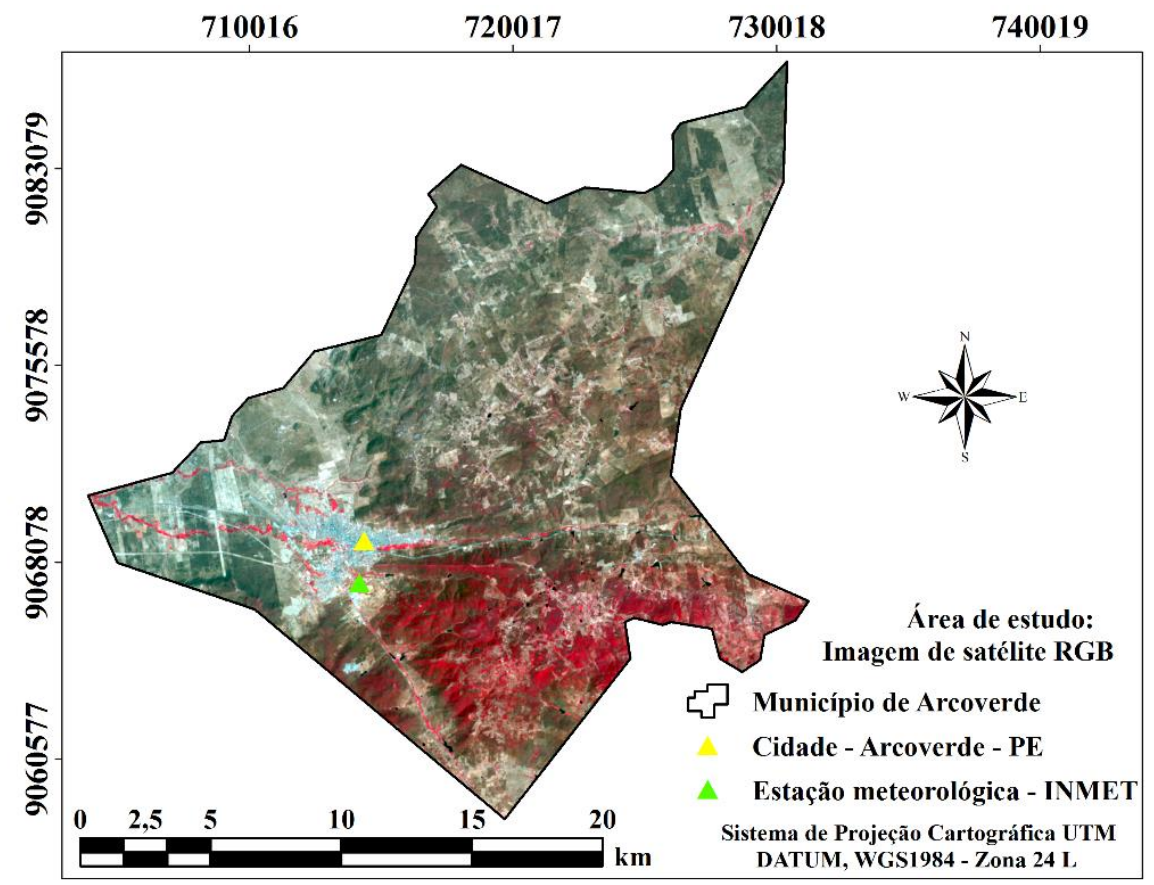

Figura 1. Mapa espacial de localização da área de estudo, município de Arcoverde, Pernambuco, Brasil. Fonte: Silva et al. (2021).

\section{Dados meteorológicos de superfície}

Foram coletados dados meteorológicos de superfície para auxiliar no processamento das imagens de satélite, tais como: temperatura do ar $\left(\mathrm{T}_{\mathrm{ar}},{ }^{\circ} \mathrm{C}\right)$, umidade relativa do ar (UR, \%), pressão atmosférica do $\operatorname{ar}\left(\mathrm{P}_{\mathrm{o}}, \mathrm{kPa}\right)$ e o ângulo zenital solar $(\theta)$, essenciais na calibração e correção das imagens (Tabela 1). Os dados foram registrados pela Instituto Nacional de 
Meteorologia (INMET) e fornecidos pela Agência Pernambucana de Águas e Climas (APAC).

Tabela 1. Dados de superfície da estação meteorológica automática localizada no município de Arcoverde-PE. Fonte: Silva et al. (2021).

\begin{tabular}{cccccc}
\hline Data & Satélite & $\boldsymbol{\theta}$ & $\mathbf{T}_{\text {ar }}\left({ }^{\circ} \mathbf{C}\right)$ & UR $(\boldsymbol{\%})$ & $\mathbf{P}_{\mathbf{o}}(\mathbf{k P a})$ \\
\hline $10 / 11 / 2008$ & \multirow{2}{*}{ Landsat-5 } & 0,88 & 23,50 & 62,00 & 82,23 \\
$13 / 11 / 2009$ & Sensor TM & 0,88 & 22,05 & 68,00 & 80,97 \\
$29 / 09 / 2010$ & & 0,87 & 22,70 & 80,00 & 81,55 \\
\hline $14 / 11 / 2015$ & Landsat-8 & 0,90 & 26,00 & 72,50 & 84,06 \\
$29 / 09 / 2016$ & Sensores & 0,90 & 24,20 & 81,00 & 82,78 \\
$05 / 12 / 2017$ & OLI/TIRS & 0,88 & 27,80 & 68,00 & 85,18 \\
\hline
\end{tabular}

\section{Dados espaciais de satélite}

A presente pesquisa foi desenvolvida a partir de seis imagens de satélites, sendo três do Landsat-5 de sensor TM (Thematic Mapper) imageadas nos dias 10/11/2008 (ano climaticamente normal), 13/11/2009, 29/09/2010 (anos climaticamente chuvosos), e três do Landsat-8 de sensores OLI (Operational Land Imager) e TIRS (Thermal Infrared Sensor) imageadas nos dias 14/11/2015, 29/09/2016 (anos climaticamente secos) e 05/12/2017 (ano climaticamente normal). Os anos estudados foram classificados climaticamente pela técnica de Quantis, como sendo anos chuvosos, normais e secos (Xavier \& Xavier, 1999). As imagens foram disponibilizadas pela agência espacial da National Aeronautics and Space Administration (NASA) e adquiridas na United States Geological Survey (USGS). Vale destacar também que em todas as imagens não foram detectadas a presença de nuvens, facilitando assim a extração de informações confiáveis da superfície terrestre da região semiárida.

As imagens foram processadas no Software ERDAS IMAGINE ${ }^{\circledR}, 9.1$, com a implementação de etapas do Surface Energy Balance Algorithms for Land (SEBAL) (Bastiaanssen, 2000; Allen et al., 2002). No algoritmo SEBAL foi realizado diante de operações matemáticas um procedimento numérico iterativo por meio das bandas multiespectrais, utilizando um sistema de modelagem espaço-temporal na caracterização de mapas temáticos da superfície terrestre dos parâmetros biofísicos albedo e temperatura da superfície, e o saldo de radiação à superfície (Bastiaanssen, 2000). O processamento digital de bandas multiespectrais das imagens de satélite se deu inicialmente pelo empilhamento de bandas e georreferencimento no Sistema de Projeção Cartográfica UTM (Universal Transversa Mercator) DATUM: WGS1984 - Zona 24 L, na qual se localiza a área do presente estudo.

Através do processamento digital das imagens, foi necessário converter os níveis de cinza das imagens de satélite em radiância espectral e refletância monocromática, isso se deu por meio de técnicas de sensoriamento remoto a partir de operações e modelagem matemática no algoritmo SEBAL. A radiância, por exemplo, representa a energia solar refletida por cada pixel das imagens por unidade de área, tempo, ângulo sólido e comprimento de onda, medida ao nível do satélite Landsat-5 TM para as bandas multiespectrais $1 ; 2 ; 3 ; 4 ; 5$ e 7 ; e do Landsat-8 OLI para as bandas multiespectrais $2 ; 3 ; 4$; 5; 6 e 7. Para o processo de conversão dos níveis de cinza para radiância e refletância do satélite Landsat-5 de sensor TM foi necessário se utilizar de coeficientes de calibração, representados pelos coeficientes de radiância mínima $\left(\mathrm{L}_{\text {mín }}\right)$ e máxima $\left(\mathrm{L}_{\text {máx }}\right)$, referente ao período após abril de 2007 para cada banda (azul; verde; vermelho; infravermelho: IV-próximo, IVmédio termal e IV-médio), além da irradiância solar espectral $\left(\mathrm{k}_{\mathrm{b}}\right.$ Land 5) de cada uma das bandas do Landsat-5 TM no topo da Atmosfera (Tabela 2).

Tabela 2. Coeficientes de calibração para conversão dos níveis de cinza das imagens de satélite em radiância e refletância para o Landsat-5 TM. Fonte: Silva et al. (2021).

\begin{tabular}{|c|c|c|c|c|}
\hline \multirow[t]{2}{*}{ Banda } & \multirow[t]{2}{*}{$\begin{array}{c}\text { Comprimento de } \\
\text { ondas }(\mu \mathrm{m})\end{array}$} & \multicolumn{2}{|c|}{$\begin{array}{l}\text { Coeficiente de calibração do } \\
\text { Landsat-5 de sensor TM } \\
\left(\mathbf{W} \mathbf{m}^{-2} \mathbf{s r}^{-1} \mu \mathrm{m}^{-1}\right)\end{array}$} & \multirow{2}{*}{$\begin{array}{c}\text { Irradiância solar espectral } \\
\left(k_{b \text { Land 5) no topo da }}\right. \\
\text { atmosfera }\left(W \mathbf{~ m}^{-2} \boldsymbol{\mu m}^{-1}\right)\end{array}$} \\
\hline & & $\mathbf{L}_{\text {mín }}$ & $\mathbf{L}_{\text {máx }}$ & \\
\hline 1 (azul) & $0,45-0,52$ & $-1,52$ & 169 & 1957 \\
\hline 2 (verde) & $0,52-0,60$ & $-2,84$ & 333 & 1796 \\
\hline 3 (vermelho) & $0,63-0,69$ & $-1,17$ & 264 & 1536 \\
\hline 4 (IV-próximo) & $0,76-0,90$ & $-1,51$ & 221 & 1031 \\
\hline 5 (IV-médio) & $1,55-1,75$ & $-0,37$ & 30,2 & 220 \\
\hline 6 (IV-termal) & $10,40-12,50$ & 1,2378 & 15,3 & - \\
\hline 7 (IV-médio) & $2,08-2,35$ & $-0,15$ & 16,0 & 83,44 \\
\hline
\end{tabular}


Deste modo, para cada banda do Landsat- 5 de sensor TM a radiância espectral foi determinada a partir da Equação 1 (Chander et al., 2009).

$\mathrm{L}_{\mathrm{b} \text { Land } 5}=\mathrm{L}_{\text {mín } \mathrm{b}}+\left(\frac{\mathrm{L}_{\text {máx } \mathrm{b}}-\mathrm{L}_{\text {min } \mathrm{b}}}{255}\right) \times\left(\mathrm{ND}_{\mathrm{b}}-1\right) \quad$ Eq. $(1)$

onde $\mathrm{Lb}$ Land $5\left(\mathrm{~W} \mathrm{~m}^{-2} \mathrm{sr}^{-1} \mu \mathrm{m}^{-1}\right)=$ radiância espectral de cada pixel em cada banda do Landsat-5, b = representa cada uma das bandas do satélite, $\mathrm{L}_{\text {mín b }} \mathrm{e}$ $\mathrm{L}_{\text {máx } \mathrm{b}}=$ radiâncias espectrais mínima e máxima $\left(\mathrm{W} \mathrm{m}^{-}\right.$ ${ }^{2} \mathrm{sr}^{-1} \mu \mathrm{m}^{-1}$ ), respectivamente (Tabela 2) e $\mathrm{ND}=$ intensidade do pixel (número digital entre 0 e 256 níveis de cinza).

Assim, também para cada banda do satélite Landsat-8 de sensor OLI foi determinada a radiância espectral a partir da Equação 2 (Chander et al., 2009; Silva et al., 2016).

$$
\mathrm{L}_{\mathrm{b} \text { Land } 8}=\mathrm{Add}_{\mathrm{rad}}+\mathrm{Mult}_{\mathrm{rad}} \times \mathrm{ND}_{\mathrm{b}} \quad \text { Eq.(2) }
$$

onde $\mathrm{L}_{\mathrm{b} \text { Land } 8}\left(\mathrm{~W} \mathrm{~m}^{-2} \mathrm{sr}^{-1} \mu \mathrm{m}^{-1}\right)=$ radiância espectral em cada banda do Landsat- 8 , baseado nos termos aditivo $\left(\mathrm{Add}_{\mathrm{rad}}\right)$ e multiplicativo (Multrad) relativos à radiância e $\mathrm{ND}=$ intensidade do pixel (número digital entre $0 \mathrm{e}$ 65.535 níveis de cinza, alto nível de detalhamento da imagem, garantindo maiores informações da área de estudo).

Através da radiância espectral, posteriormente foi determinada a refletância monocromática para cada banda das imagens do satélite Landsat-5 de sensor TM, baseada na irradiância solar espectral no topo da Atmosfera ( $\mathrm{k}_{\mathrm{b} \text { Land 5}}$, Tabela 2) conforme a Equação 3 (Chander et al., 2009; Silva et al., 2016).

$\mathrm{r}_{\mathrm{b} \text { Land } 5}=\frac{\pi \times \mathrm{L}_{\mathrm{b} \text { Land } 5}}{\mathrm{k}_{\mathrm{b} \text { Land } 5} \times \cos \theta \times \mathrm{d}_{\mathrm{r}}}$

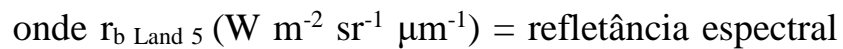
monocromática em cada banda do Landsat-5 TM, $\theta=$ ângulo zenital solar, obtido através do ângulo de elevação do Sol, fornecido pela NASA/USGS e $d_{r}=$ quadrado da razão entre as distâncias média e instantânea entre a Terra e o Sol em dado dia do ano imageado.

Assim, também foi determinada a refletância para cada banda do Landsat-8 OLI conforme a Equação 4 (Chander et al., 2009; Silva et al., 2016).

$$
\mathrm{r}_{\mathrm{b} \text { Land } 8}=\frac{\left(\mathrm{Add}_{\mathrm{ref}}+\mathrm{Mult}_{\mathrm{ref}} \times \mathrm{ND}_{\mathrm{b}}\right)}{\cos \theta \times \mathrm{d}_{\mathrm{r}}}
$$

onde $\mathrm{r}_{\mathrm{b}}$ Land $8\left(\mathrm{~W} \quad \mathrm{~m}^{-2} \mathrm{sr}^{-1} \quad \mu \mathrm{m}^{-1}\right)=$ refletância monocromática em cada banda do Landsat-8 OLI, também com base nos termos aditivo $\left(\operatorname{Add}_{\mathrm{ref}}\right) \mathrm{e}$ multiplicativo (Mult ref ), relativos à refletância.
O quadrado da razão entre as distâncias média e instantânea entre a Terra e o Sol foi determinado a partir da Equação 5 (Iqbal, 1983).

$$
\mathrm{d}_{\mathrm{r}}=1+0,033 \times \cos \left(\frac{\mathrm{DSA} \times 2 \times \pi}{365}\right)
$$

onde DSA = dia sequencial do ano; em que o argumento da função cos está em radianos.

O ângulo zenital solar $(\theta)$ foi determinado a partir do ângulo de elevação do sol para todas as imagens do presente estudo, a partir da Equação 6.

$$
\theta=\cos \left(\frac{\pi}{2}-E\right)=\operatorname{sen}(E) \quad \text { Eq. }(6)
$$

No presente estudo também foram determinados os próprios coeficientes de pesos $\left(\mathrm{p}_{\mathrm{b}}\right)$ das bandas refletivas das imagens do satélite Landsat8 de sensor OLI conforme a Equação 7, necessários para o computo do albedo planetário. Os pesos foram calculados por meio da irradiância solar espectral $\left(\mathrm{k}_{\mathrm{b}}\right.$, $\mathrm{W} \mathrm{m}^{-2} \mu \mathrm{m}^{-1}$ ) de cada banda do Landsat-8 OLI a partir da Equação 8 (Chander \& Markham, 2003; Silva et al., 2016).

$$
\begin{aligned}
& \mathrm{k}_{\mathrm{b}}=\frac{\pi \times \mathrm{L}_{\mathrm{b}}}{\mathrm{r}_{\mathrm{b}} \times \cos \theta \times \mathrm{d}_{\mathrm{r}}} \\
& \mathrm{p}_{\mathrm{b}}=\frac{\mathrm{k}_{\mathrm{b}}}{\Sigma \mathrm{k}_{\mathrm{b}}}
\end{aligned}
$$

Sendo assim, foi empregado para a estimativa do albedo planetário (topo da Atmosfera), os valores dos respectivos pesos encontrados anteriormente para cada banda dos satélites conforme a Equação 9 (Silva et al., 2016).

$$
\begin{aligned}
& \alpha_{\text {toa }}=\mathrm{p}_{1,2} \times \mathrm{r}_{1,2}+\mathrm{p}_{2,3} \times \mathrm{r}_{2,3}+\mathrm{p}_{3,4} \times \mathrm{r}_{3,4}+\mathrm{p}_{4,5} \times \mathrm{r}_{4,5}+ \\
& \mathrm{p}_{5,6} \times \mathrm{r}_{5,6}+\mathrm{p}_{6,7} \times \mathrm{r}_{6,7}
\end{aligned}
$$

onde $\alpha_{\mathrm{toa}}=$ albedo planetário, $\mathrm{p}_{1,2}-\mathrm{p}_{6,7}=$ respectivos pesos de cada uma das bandas dos satélites; $r_{1,2} ; r_{2,3}$, $r_{3,4}, r_{4,5}, r_{5,6}$ e $r_{6,7}=$ respectivas refletâncias do Landsat5 TM e Landsat- 8 OLI.

Um dos principais objetivos do presente estudo foi determinar o albedo da superfície, corrigido para os efeitos atmosféricos, conforme Equação 10 (Silva et al., 2016).

$$
\alpha_{\text {sup }}=\frac{\alpha_{\text {toa }}-\alpha_{\text {atm }}}{\tau_{\mathrm{sw}}^{2}}
$$

onde $\alpha_{\text {sup }}=$ albedo da superfície corrigido, $\alpha_{\text {atm }}=$ refletância da própria atmosfera (Allen et al., 2002) e 
$\tau_{\mathrm{sw}}=$ transmissividade atmosférica no domínio da radiação solar, conforme a Equação 11 (Allen et al., 2007; Silva et al., 2016).

$\tau_{\mathrm{sW}}=0,35+0,627 \times \exp \left[\frac{-0,00146 \times \mathrm{P}_{\mathrm{o}}}{\mathrm{K}_{\mathrm{t}} \times \cos \theta}-0,075\left(\frac{\mathrm{W}}{\cos \theta}\right)^{0,4}\right]$ Eq. $(11)$

onde $\mathrm{P}_{\mathrm{o}}(\mathrm{kPa})=$ pressão atmosférica instantânea, $\mathrm{K}_{\mathrm{t}}=$ coeficiente de turbidez da atmosfera $\left(K_{t}=1,0\right.$, para um dia com céu claro), de acordo com Allen et al. (2002) e Silva et al. (2016), W (mm) = água precipitável, estimada a partir da Equação 12 (Garrison \& Adler, 1990).

$$
\mathrm{W}=0,14 \times \mathrm{e}_{\mathrm{a}} \times \mathrm{P}_{\mathrm{o}}+2,1
$$

onde $\mathrm{e}_{\mathrm{a}}(\mathrm{kPa})=$ pressão real de vapor d'água atmosférico, estimada a partir da Equação 13.

$$
\mathrm{e}_{\mathrm{a}}=\frac{\mathrm{UR} \times \mathrm{e}_{\mathrm{s}}}{100}
$$

onde UR $(\%)=$ umidade relativa do ar instantânea, $\mathrm{e}_{\mathrm{s}}$ $(\mathrm{kPa})=$ pressão de saturação do vapor d'água, estimada a partir da Equação 14.

$$
\mathrm{e}_{\mathrm{s}}=0,6108 \times \exp \left(\frac{17,27 \times \mathrm{T}_{\mathrm{ar}}}{237,3+\mathrm{T}_{\mathrm{ar}}}\right)
$$

onde $\mathrm{T}_{\text {ar }}\left({ }^{\circ} \mathrm{C}\right)=$ temperatura do ar instantânea, no momento da passagem do satélite (Tabela 1).

Também a partir das bandas refletivas das imagens dos satélites foram determinados os índices de vegetação, tais como, o índice de vegetação ajustado ao solo (Soil Adjusted Vegetation Index SAVI), Equação 15 (Allen et al., 2002).

$$
\mathrm{SAVI}=\frac{(1+\mathrm{L}) \times\left(\mathrm{r}_{\mathrm{bIV}}-\mathrm{r}_{\mathrm{bV}}\right)}{\left(\mathrm{L}+\mathrm{r}_{\mathrm{bIV}}+\mathrm{r}_{\mathrm{bV}}\right)}
$$

onde $\mathrm{r}_{\mathrm{b}}$ Iv e $\mathrm{r}_{\mathrm{b}} \mathrm{v}=$ bandas refletivas 4 e 3 do Landsat -5 de sensor TM e bandas 5 e 4 do Landsat- 8 de sensor OLI, respectivamente, $\mathrm{L}=$ fator de ajuste ao solo, que varia entre 0 e 1 , de acordo com a densidade da cobertura vegetal da região de estudo. Tal qual foi utilizado o fator de ajuste de 0,5 , este que indica uma área com cobertura vegetal intermediária (Huete, 1988; Allen et al., 2007). O fator escolhido faz referência a área de estudo que é localizada no semiárido brasileiro e possui vegetação de Caatinga (Silva \& Galvíncio, 2012).

Através do índice de vegetação SAVI, também foi determinado o índice de área foliar (IAF, $\mathrm{m}^{2} \mathrm{~m}^{-2}$ ), conforme a Equação 16 (Allen et al., 2007).

$$
\mathrm{IAF}=-\frac{\ln \left(\frac{0,69-\mathrm{SAVI}}{0,59}\right)}{0,91}
$$

A partir do IAF foi determinada a emissividade no domínio espectral da banda termal $\left(\varepsilon_{\mathrm{NB}}\right)$, esta que é essencial na determinação da temperatura da superfície, Equações 17 (Allen et al., 2002).

$$
\varepsilon_{\mathrm{NB}}=0,97+0,0033 \times \mathrm{IAF}
$$

No intuito de determinar o saldo de radiação à superfície (balanço de radiação), foi realizado o cômputo da radiação de onda longa emitida, considerando deste modo a emissividade no domínio da banda larga $\left(\varepsilon_{0}\right)$ determinada de acordo com a Equação 18 (Allen et al., 2002).

$$
\varepsilon_{0}=0,95+0,01 \times \mathrm{IAF}
$$

A temperatura da superfície, em Kelvin, foi determinada conforme a Equação 19 (Allen et al., 2002).

$$
\mathrm{T}_{\mathrm{s}}=\frac{\mathrm{K}_{2}}{\left(\frac{\varepsilon_{\mathrm{NB}} \times \mathrm{K}_{1}}{\mathrm{~L}_{\mathrm{b}}}+1\right)}
$$

onde $\mathrm{K}_{1}$ e $\mathrm{K}_{2}=$ constantes de calibração da banda termal dos satélites Landsat-5 de sensor TM (banda termal 6: $\mathrm{K}_{1}=607,76 \mathrm{~W} \mathrm{~m}^{-2} \mathrm{sr}^{-1} \mu \mathrm{m}^{-1}$ e $\mathrm{K}_{2}=1260,56$ $\mathrm{K})$ e Landsat- 8 de sensor TIRS (banda termal 10: $\mathrm{K}_{1}=$ $774,89 \mathrm{~W} \mathrm{~m}^{-2} \mathrm{sr}^{-1} \mu \mathrm{m}^{-1}$ e $\mathrm{K}_{2}=1321,08 \mathrm{~K}$ ), fornecidas pela NASA/USGS.

Por fim, foi estimado o saldo de radiação à superfície terrestre (balanço de radiação) conforme a Equação 20 (Allen et al., 2002; Oliveira et al., 2015).

$$
\mathrm{R}_{\mathrm{n}}=\mathrm{R}_{\text {sol inc } \downarrow} \times\left(1-\alpha_{\text {sup }}\right)-\mathrm{R}_{\text {ol emit } \uparrow}+\mathrm{R}_{\text {ol atm } \downarrow}-\left(1-\varepsilon_{0}\right) \times \mathrm{R}_{\text {ol atm } \downarrow}
$$

onde $\mathrm{R}_{\mathrm{n}}\left(\mathrm{W} \mathrm{m}^{-2}\right)=$ saldo de radiação à superfície, $\mathrm{R}_{\mathrm{sol}}$ inc $\downarrow$ radiação de onda curta incidente; $\alpha_{\text {sup }}$ - albedo corrigido, $R_{\mathrm{ol} \mathrm{atm} \downarrow}=$ radiação de onda longa emitida pela atmosfera e $R_{\text {ol emit } \uparrow}$ = radiação de onda longa emitida.

\section{Análise estatística}

Os parâmetros biofísicos foram analisados através da estatística descritiva, por meio de medidas de tendência central (média) e dispersão (mínimo, máximo, desvio padrão - DP e coeficiente de variação - CV). Os mapas temáticos foram avaliados quanto a sua variabilidade espaço-temporal a partir dos valores do coeficiente de variação $(\mathrm{CV}, \%)$ conforme os critérios de classificação de Warrick \& Nielsen (1980): CV $<12 \%$ - caracterizado de baixa 
variabilidade; entre $12 \%<\mathrm{CV}<60 \%$ - média variabilidade; e CV $>60 \%$ - alta variabilidade.

\section{Resultados e Discussão}

O monitoramento espaço-temporal através de mapas temáticos da superfície terrestre originou padrões de distribuição satisfatório da vegetação de Caatinga e demais usos do solo no município de Arcoverde-PE, entre o período de 2008 a 2017. As análises possibilitaram enfatizar a diferenciação de áreas conforme o seu uso e ocupação do solo, deixando evidenciadas áreas degradadas e/ou que precisam de recuperação e atenção, são mudanças ambientais provocadas pelo efeito da seca e/ou pelas atividades antrópicas praticadas na região semiárida.
Com isso, foi possível a identificação de áreas de solo exposto, corpos hídricos, área urbana e de vegetação relativamente densa.

Os mapas temáticos dos parâmetros biofísicos do balanço de energia apresentaram uma distribuição espaço-temporal de média variabilidade ( $\mathrm{CV}$ variando entre 23,00 e $27,00 \%$, Tabela 3) para o albedo da superfície e alta variabilidade (CV variando entre 6,48 e $12,00 \%$, Tabelas 4 e 5 , respectivamente) para a temperatura e o saldo de radiação à superfície (Warrick \& Nielsen, 1980).

Na Figura 2 está ilustrado o mapa temático do albedo da superfície, destacando a distribuição espaço-temporal da dinâmica da cobertura vegetal de Caatinga densa e rala e áreas irrigadas.



Figura 2. Distribuição espaço-temporal do albedo da superfície na região semiárida de Arcoverde-PE. Fonte: Silva et al. (2021).

As imagens foram classificadas em seis subgrupos, como ilustrado nos mapas temáticos, facilitando deste modo o diagnóstico das áreas preservadas e degradadas. Todavia, os mapas destacam os distintos usos e ocupações do solo, apresentando mudanças ao longo do tempo que podem influenciar no clima local da região. Os mapas evidenciam áreas preservadas na região centro-sul (pixels de tonalidades azul), e as áreas com solo exposto dentre todo o município de Arcoverde (pixels de tonalidades vermelhas) (Figura 2).
Os mapas temáticos do albedo obtiveram valores variando entre 0,04 e 0,55 . Os menores valores, principalmente próximos de zero $(0,04)$, foram observados sobre os corpos hídricos, sendo as áreas com vegetação de Caatinga densa e áreas irrigadas de valores entre 0,04 e 0,15 . Por outro lado, os maiores valores foram observados sobre a área urbana e também nas áreas de solo exposto, tal qual foram áreas que detectaram valores do albedo entre 0,19 e 0,55 . A faixa de valor entre 0,16 e 0,18 foram áreas de vegetação de Caatinga rasteira (Figura 2). 
Na Tabela 3 destaca-se os parâmetros estatísticos e a variabilidade quantitativa e espaço- temporal do albedo da superfície para a região semiárida de Arcoverde-PE.

Tabela 3. Parâmetros estatísticos e variabilidade quantitativa e espaço-temporal do albedo da superfície. Fonte: Silva et al. (2021).

\begin{tabular}{lcccccc}
\hline \multirow{2}{*}{ Data } & Classificação & \multicolumn{5}{c}{ Albedo da superfície } \\
\cline { 3 - 7 } & dos anos & Mínimo & Máximo & Médio & DP & CV (\%) \\
\hline $10 / 11 / 2008$ & Normal & 0,04 & 0,64 & 0,16 & 0,03 & 23,00 \\
$13 / 11 / 2009$ & Chuvoso & 0,04 & 0,55 & 0,15 & 0,03 & 24,00 \\
$29 / 09 / 2010$ & Chuvoso & 0,03 & 0,68 & 0,14 & 0,03 & 23,00 \\
$14 / 11 / 2015$ & Seco & 0,03 & 0,69 & 0,22 & 0,06 & 27,00 \\
$29 / 09 / 2016$ & Seco & 0,04 & 0,83 & 0,20 & 0,05 & 27,00 \\
$05 / 12 / 2017$ & Normal & 0,02 & 0,71 & 0,18 & 0,04 & 26,00 \\
\hline
\end{tabular}

Vale destacar que os maiores valores médios do albedo foram verificados em anos climaticamente secos, como nos dias 14/11/2015 e 29/09/2016, com valores respectivos de 0,22 e 0,20 , seguido dos anos climaticamente normais, como nos dias 10/11/2008 e 05/12/2017, com valores de 0,16 e 0,18 , respectivamente, destacando o efeito severo das épocas de seca na região semiárida. Os anos climaticamente chuvosos foram os que tiveram os menores valores médios do albedo, com valores de $0,15(13 / 11 / 2009)$ e $0,14(29 / 09 / 2010)$, provavelmente devido à sua maior quantidade e densidade de vegetação de Caatinga (Tabela 3 ).

Os valores do albedo são coerentes e refletem a ligação proporcional entre o aumento das áreas de solo exposto e a elevação do albedo, provocada pelos eventos de seca e escassez hídrica e, ainda, pelo manejo inadequado do uso do solo, sendo o albedo um parâmetro adequado para o monitoramento das mudanças ambientais, as quais podem favorecer a degradação e a desertificação, como destacado em diversos estudos, especialmente no semiárido brasileiro (Arraes et al., 2012; Bezerra et al., 2014, Giongo \& Vettorazzi, 2014; Oliveira et al., 2015; Lins et al, 2017; Silva et al., 2019).

A Figura 3 apresenta os mapas temáticos da temperatura da superfície. A distribuição espaçotemporal dos mapas destaca o padrão de comportamento da temperatura em função dos diferentes usos do solo no município de ArcoverdePE.

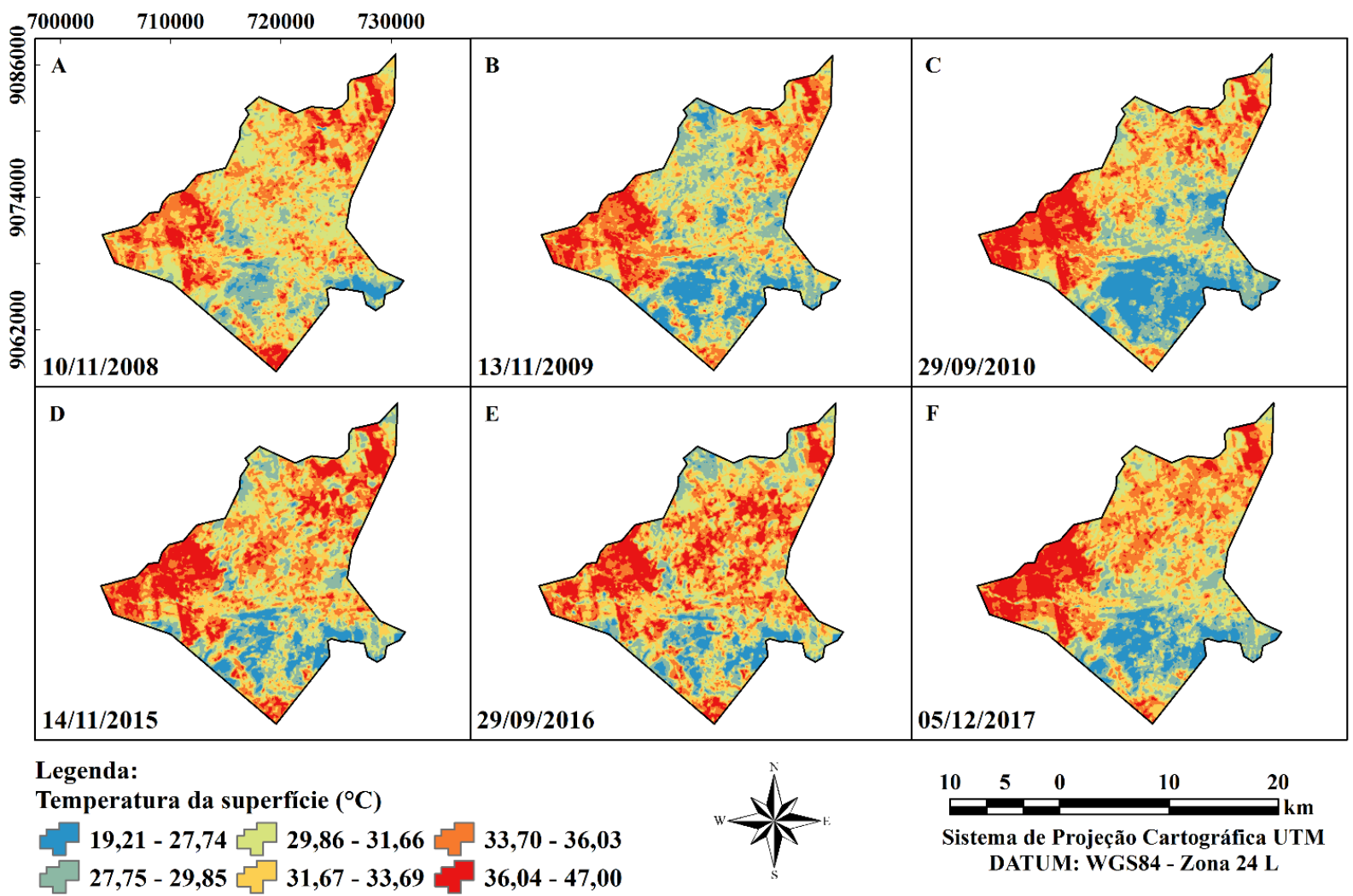

Figura 3. Distribuição espaço-temporal da temperatura da superfície na região semiárida de Arcoverde-PE. Fonte: Silva et al. (2021). 
As maiores temperaturas se concentraram nas regiões nordeste, norte e oeste dos mapas temáticos, demonstrando também que as áreas da região centrosul possuem maior densidade de vegetação, que por sua vez contribuem substancialmente para a diminuição da temperatura da superfície na região, devido a capacidade do dossel em absorver radiação e impedir que essa mesma radiação chegue ao solo e se transforme em ondas curtas, aquecendo substancialmente a área, o que melhora diversos aspectos da superfície (Figura 3).

Os mapas da temperatura alcançaram valores entre $19,21^{\circ} \mathrm{C}$ e $47^{\circ} \mathrm{C}$, seguindo basicamente o mesmo comportamento de distribuição do albedo na análise espaço-temporal. Sendo os menores valores encontrados principalmente nos corpos hídricos, áreas irrigadas e bem vegetadas, entre $19,21^{\circ} \mathrm{C}$ e $27,74^{\circ} \mathrm{C}$ (pixels de tonalidade azul). Os maiores valores foram encontrados na área urbana e principalmente sobre áreas de solo exposto, com valores variando entre $31,67^{\circ} \mathrm{C}$ e $47^{\circ} \mathrm{C}$ (Figura 3 ).

$\mathrm{Na}$ Tabela 4 destaca-se os parâmetros estatísticos e a variabilidade quantitativa e espaçotemporal da temperatura da superfície para a região semiárida de Arcoverde-PE.

Tabela 4. Parâmetros estatísticos e variabilidade quantitativa e espaço-temporal da temperatura da superfície. Fonte: Silva et al. (2021).

\begin{tabular}{lcccccc}
\hline \multirow{2}{*}{ Data } & Classificação & \multicolumn{5}{c}{ Temperatura da superfície $\left({ }^{\circ} \mathbf{C}\right)$} \\
\cline { 3 - 7 } & dos anos & Mínimo & Máximo & Médio & DP & CV (\%) \\
\hline $10 / 11 / 2008$ & Normal & 19,21 & 42,06 & 32,08 & 2,77 & 9,00 \\
$13 / 11 / 2009$ & Chuvoso & 22,40 & 41,53 & 31,70 & 2,99 & 9,00 \\
$29 / 09 / 2010$ & Chuvoso & 23,70 & 43,45 & 33,49 & 3,89 & 12,00 \\
$14 / 11 / 2015$ & Seco & 30,00 & 47,00 & 39,00 & 3,13 & 8,00 \\
$29 / 09 / 2016$ & Seco & 28,00 & 43,90 & 37,70 & 2,50 & 7,00 \\
$05 / 12 / 2017$ & Normal & 28,00 & 46,00 & 38,00 & 3,23 & 9,00 \\
\hline
\end{tabular}

O dia 14/11/2015 (ano seco) apresentou o maior valor médio da temperatura, de $47^{\circ} \mathrm{C}$. Destacando assim como o albedo, o efeito severo da seca na região semiárida. Contudo, a baixa regeneração da cobertura vegetal de Caatinga pela falta de chuva, favorece a escassez de água e o aumento da temperatura da superfície (Aquino et al., 2016; Coutinho et al., 2016). O dia 13/11/2009 (ano chuvoso) se destacou pela menor temperatura média, de $41,53^{\circ} \mathrm{C}$ (Tabela 4). Os eventos de chuva favorecem a resiliência da vegetação de Caatinga no semiárido, sendo que o maior aporte de umidade junta a vegetação mais adensada favorece um microclima local na região (Arraes et al., 2012; Bezerra et al., 2014).

Na Figura 4 está ilustrado os mapas temáticos do saldo de radiação à superfície. A distribuição espaço-temporal enfatiza o padrão de resposta do saldo de radiação nos diferentes usos do solo do município de Arcoverde-PE.

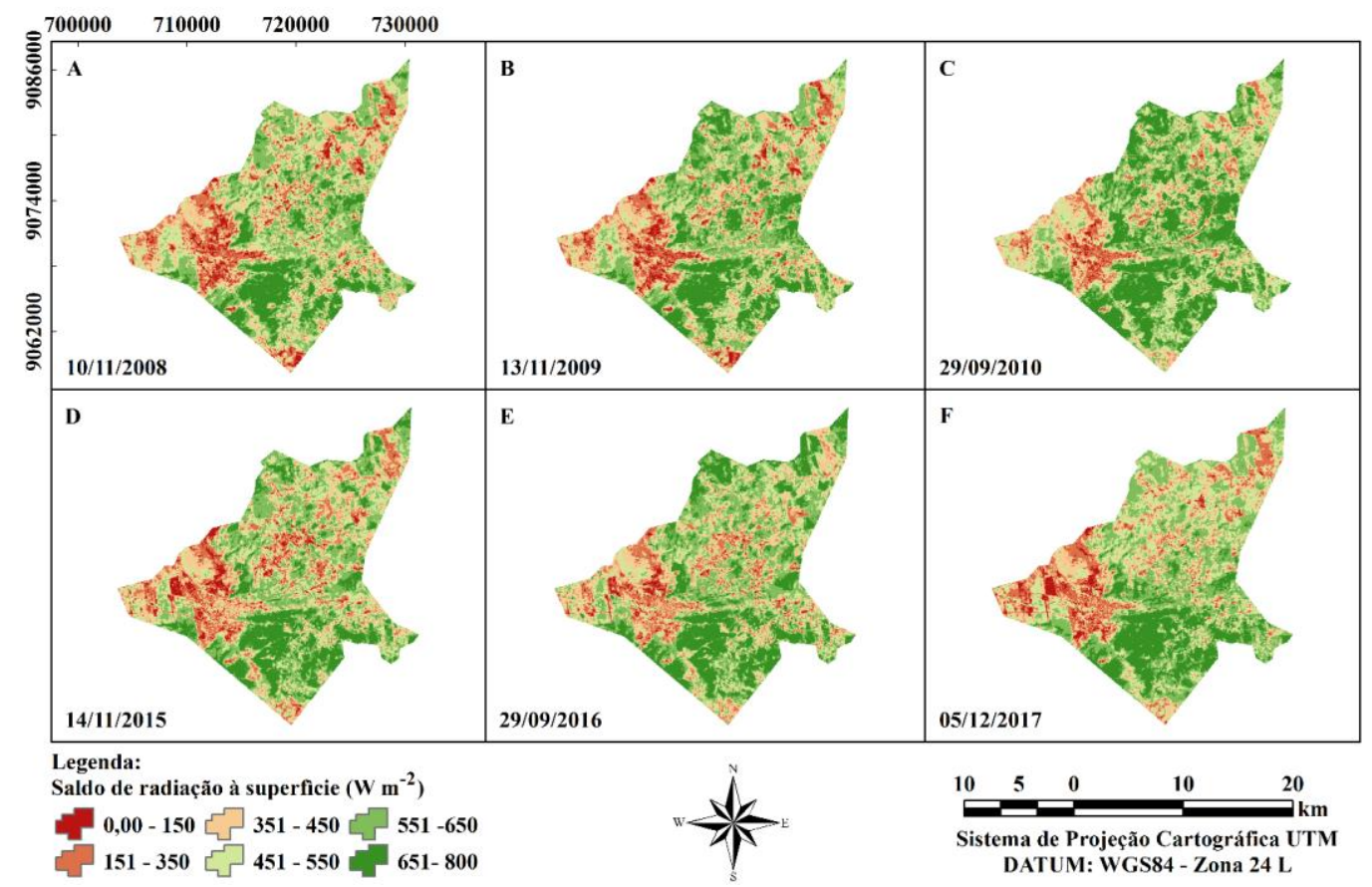

Figura 4. Distribuição espaço-temporal do saldo de radiação à superfície na região semiárida de Arcoverde-PE. Fonte: Silva et al. (2021). 
Os mapas do saldo de radiação obtiveram valores variando entre 0,0 e $800 \mathrm{~W} \mathrm{~m}^{-2}$. Os maiores valores se concentraram nas regiões das áreas centrosul dos mapas temáticos, justamente as regiões que tiveram os menores valores do albedo e temperatura, por serem áreas que possuem vegetação mais densa, com baixo déficit hídrico e, também, por estas regiões comporem áreas irrigadas, favorecendo deste modo, uma repartição de energia de forma equilibrada na região (Figura 3). Estas são áreas resultantes de menor reflexão pelas ondas curtas, ocorrendo assim menor emissão das ondas longas (Oliveira et al., 2015).

Os menores valores foram encontrados principalmente sobre as áreas de solo exposto e área urbana da região (Figura 4), estas que tiveram ao mesmo tempo, os maiores valores do albedo $\mathrm{e}$ temperatura, que se justificam por serem regiões com perda de energia solar, tanto por emissão quanto por reflexão (Oliveira et al., 2015). Estas áreas apresentaram valores mínimos de até 0,0 e $36 \mathrm{~W} \mathrm{~m}^{-2}$ (Tabela 5), devido serem anos climaticamente secos para a região. Arraes et al. (2012) identificaram as menores médias do saldo sobre área antrópica, assim, destacaram uma menor existência de energia disponível a ser repartida no processo da evapotranspiração, aquecimento do ar e aquecimento do solo.

A Tabela 5 destaca os parâmetros estatísticos e a variabilidade quantitativa e espaço-temporal do saldo de radiação à superfície para a região semiárida de Arcoverde-PE.

Tabela 5. Parâmetros estatísticos e variabilidade quantitativa e espaço-temporal do saldo de radiação à superfície. Fonte: Silva et al. (2021).

\begin{tabular}{|c|c|c|c|c|c|c|}
\hline \multirow{2}{*}{ Data } & \multirow{2}{*}{$\begin{array}{c}\text { Classificação } \\
\text { dos anos }\end{array}$} & \multicolumn{5}{|c|}{ Saldo de radiação à superfície $\left(\mathrm{W} \mathbf{m}^{-2}\right)$} \\
\hline & & Mínimo & Máximo & Médio & DP & $\mathrm{CV}(\%)$ \\
\hline $10 / 11 / 2008$ & Normal & 106 & 690 & 624 & 46 & 7,37 \\
\hline $13 / 11 / 2009$ & Chuvoso & 254 & 800 & 642 & 48 & 7,48 \\
\hline $29 / 09 / 2010$ & Chuvoso & 120 & 757 & 633 & 41 & 6,48 \\
\hline $14 / 11 / 2015$ & Seco & 36 & 655 & 550 & 73 & 13,27 \\
\hline $29 / 09 / 2016$ & Seco & 0,0 & 618 & 553 & 60 & 10,85 \\
\hline $05 / 12 / 2017$ & Normal & 86 & 675 & 582 & 60 & 10,31 \\
\hline
\end{tabular}

O dia 13/11/2009 (ano chuvoso) apresentou o maior valor médio do saldo de radiação, de $800 \mathrm{~W} \mathrm{~m}^{-2}$. Arraes; Andrade; Silva (2012) identificaram as maiores médias do saldo na área de vegetação nativa densa, favorecendo a uma maior quantidade de energia disponível a ser repartida no processo da evapotranspiração, aquecimento do ar e aquecimento do solo.

Os resultados da análise espaço-temporal destacaram que a dinâmica de distribuição do saldo de radiação à superfície está diretamente relacionada com a dinâmica e padrões de respostas do albedo e temperatura da superfície. As regiões com os menores valores do albedo e temperatura apresentaram os maiores valores do saldo para todo o período estudado.

A variabilidade quantitativa e espaçotemporal da cobertura vegetal de Caatinga e demais usos do solo da região apresentaram através de mapas temáticos dos parâmetros biofísicos o padrão de resposta das mudanças ambientais da região semiárida para compreensão e investigação do ambiente natural e degradado, atuando como instrumento para conservação do bioma Caatinga no semiárido brasileiro.

\section{Conclusões}

$\mathrm{O}$ aumento dos índices biofísicos albedo e temperatura da superfície destacaram mudanças nos diferentes usos do solo, evidenciando principalmente áreas de solo exposto, especialmente nos anos classificados como climaticamente secos. O monitoramento espaçotemporal do albedo e temperatura confirmaram ser bons indicadores das mudanças ambientais no semiárido.

Quando os eventos de seca se juntam as práticas intensivas de uso e ocupação do solo as consequências ao longo do tempo podem se concretizar na degradação ambiental da região, todavia, vale ressaltar também que devido à maior radiação solar nas regiões semiáridas do Brasil em determinados meses do ano, como na estação seca, o cenário vegetativo é influenciado negativamente favorecendo a elevação destes parâmetros até a estação chuvosa quando ocorre a resiliência da vegetação natural de Caatinga.

Desse modo, verifica-se que o saldo de radiação mostrou sensibilidade nos diferentes usos do solo, se destacando principalmente nas áreas irrigadas e de vegetação densa, quando houve maior energia disponível a ser repartida pelos processos do balanço de energia à superfície e evapotranspiração. A distribuição espaço-temporal e os padrões de respostas do saldo de radiação também confirmou está intensamente conexo com os padrões de comportamento do albedo e temperatura da superfície. 


\section{Agradecimentos}

À Universidade Federal Rural de Pernambuco; Programa de Pós-Graduação em Engenharia Agrícola; Departamento de Agronomia; O presente trabalho foi realizado com apoio da Coordenação de Aperfeiçoamento de Pessoal de Nível Superior - Brasil (CAPES) Código de Financiamento 001; Conselho Nacional de Desenvolvimento Científico e Tecnológico (CNPq); NASA/USGS pelo fornecimento dos dados espaciais de satélite; Instituto Nacional de Meteorologia (INMET) e Agência Pernambucana de Águas e Climas (APAC) pelos dados meteorológicos de superfície.

\section{Referências}

Allen, R. G.; Tasumi, M.; Trezza, R. 2007. Satellite-Based energy balance for mapping evapotranspiration with internalized calibration (METRIC) - Model. Journal of Irrigation and Drainage Engineering, 133, 380-394.

Alvares, C. A.; Stape, J. L.; Sentelhas, P. C.; Moraes, G.; Leonardo, J.; Parovek, G. 2013. Köppen's climate classification map for Brazil. Meteorologische Zeitschrift, 22, 711728.

Arraes, F. D. D.; Andrade, E. M.; Silva, B. B. 2012. Dinâmica do balanço de energia sobre o açude Orós e suas adjacências. Revista Caatinga, 25, 119-127.

Ballén, L. A.; Souza, B. I.; Lima, E. R. 2016. Análise espaço-temporal da cobertura vegetal na área de proteção ambiental do Cariri, Paraíba, Brasil. Boletim Goiano de Geografia, 36, 555-571.

Bastiaanssen, W. G. M.; Menenti, M.; Feddes, R. A.; Holtslag, A. A. M. 1998. A remote sensing surface energy balance algorithm for land (SEBAL) 2. Validation. Journal of Hydrology, 212-213, 213-229.

Beltrão, B. A.; Mascarenhas, J. C.; Miranda, J. L. F.; Souza Junior, L. C.; Galvão, M. J. T. G.; Pereira, S. N. 2005. Projeto cadastro de fontes de abastecimento por água subterrânea no estado de Pernambuco - Diagnóstico do município de Arcoverde. Recife: CPRM/PRODEEM, $11 \mathrm{p}$.

Bezerra, J. M.; Moura, G. B. A.; Silva, B. B.; Lopes, P. M. O.; Silva, E. F. F. 2014. Parâmetros biofísicos obtidos por sensoriamento remoto em região semiárida do estado do Rio Grande do Norte, Brasil1. Revista Brasileira de Engenharia Agrícola e Ambiental, 18, 73-84.

Chander, G.; Markham, B. L.; Helder, D. L. 2009. Summary of current radiometric calibration coefficients for Landsat MSS, TM, ETM+, and EO-1 ALI sensors. Remote Sensing of Environment, 113, 893-903.

Chander, G.; Markhan, B. 2003. Revised Landsat 5 - TM radiometric calibration procedures and post calibration dynamic ranges. IEEE Transactions on Geosciences and Remote Sensing, 41, 2674-2677.

Coutinho, M. A.; Fernandes, A. C.; Santos, V. G.; Nascimento, C. R. 2016. Análise comparativa dos índices de vegetação NDVI, SAVI, RATIO E IAF para identificação de queimadas. Caderno de Ciências Agrárias, 8, 70-81.

Garrison, J. D.; Adler, G. P. 1990. Estimation of precipitable water over the United State for aplication to the division of solar radiation into its direct and difuse coponents. Solar Energy, 44, 225-241.

Gutiérrez, A. P. A.; Engle, N. L.; De Nys, E.; Molejón, C.; Martins, E. S. 2014. Drought preparedness in Brazil. Weather and Climate Extremes, 3, 95-106.

IBGE. 2019. Instituto Brasileiro De Geografia E Estatística. Divisão Territorial do Brasil e Limites Territoriais. Disponível em: <ftp://geoftp.ibge.gov.br/organizacao_do_ter ritorio/estrutura_territorial/divisao_territorial /2016/> Acesso: 16 de julho de 2019.

Lins, F. A. C.; Araújo, D. C. S.; Silva, J. L. B.; Lopes, P. M. O.; Oliveira, J. D. A.; Silva, A. T. C. S. G. 2017. Estimativa de parâmetros biofísicos e evapotranspiração real no semiárido Pernambucano utilizando sensoriamento remoto. Irriga, 1, 64-75.

Marengo, J. A.; Alves, L. M.; Alvala, R.; Cunha, A. P.; Brito, S.; Moraes, O. L. 2018. Climatic characteristics of the 2010-2016 drought in the semiarid Northeast Brazil region. Anais da Academia Brasileira de Ciências, 90, 1973-1985.

Mariano, D. A.; Dos Santos, C. A.; Wardlow, B. D.; Anderson, M. C.; Schiltmeyer, A. V.; Tadesse, T.; Svoboda, M. D. 2018. Use of remote sensing indicators to assess effects of drought and human-induced land degradation on ecosystem health in Northeastern Brazil. Remote Sensing of Environment, 213, 129143.

Meneses, P. R. 2012. Definição de Sensoriamento Remoto. In: Meneses, P. R.; Almeida, T (Org.). Introdução ao processamento de imagens de sensoriamento remoto. Brasília: CNPq, UNB. Cap. 1, 11-33.

Oliveira, L. M. M.; Montenegro, S. M. G. L.; Silva, B. B.; Moura, A. E. S. S. 2015. Balanço de radiação por sensoriamento remoto em bacia 
hidrográfica da zona da mata nordestina. Revista Brasileira de Meteorologia, 30, 1628.

Silva, B. B. D.; Braga, A. C.; Braga, C. C.; Oliveira, L. M.; Montenegro, S. M.; Barbosa Junior, B. 2016. Procedures for calculation of the albedo with OLI-Landsat 8 images: Application to the Brazilian semi-arid. Revista Brasileira de Engenharia Agrícola e Ambiental, 20, 3-8.

Silva, D. A. O.; Lopes, P. M. O.; Moura, G. B. A.; França E Silva, E. F.; Silva, J. L. B.; Bezerra, A. C. 2019. Evolução Espaço-Temporal do Risco de Degradação da Cobertura Vegetal de Petrolina-PE. Revista Brasileira de Meteorologia, 34, 1-11.

Silva, J. L. B.; Santos Araújo, D. C.; Santos, D. P.; Bezerra, A. C.; Albuquerque Moura, G. B.; Oliveira, P. M. L.; Silva, H. P. 2017. Spatial distribution of biophysical parameters in the Ipanema River Basin using remote sensing techniques. Journal of Hyperspectral Remote Sensing, 7, 324-333.

Warrick, A. W.; Nielsen, D. R. Spatial variability of soil physical properties in the field. In: Hillel, D., ed. Applications of soil physics. Academic Press, 1980. 350 p.

Iqbal, M. 1983. An introduction to solar radiation. London: Academic Press., 390 p.

Huete, A. R. 1988. A soil adjusted vegetation index (SAVI). Remote Sensing of Environment, 25, 295-309.

Xavier, T. M. B. S; Xavier, A. F. S. 1999. Caracterização de períodos secos ou excessivamente chuvosos no estado do Ceará através da técnica dos Quantis: 1964-1998. Revista Brasileira de Meteorologia, 14, 6378.

Bastiaanssen, W. G. M. 2000. SEBAL - Based Sensible and Latent Heat Fluxes in the
Irrigated Gediz Basin, Turkey. Journal of Hidrology, 229, 87-100.

Allen, R. G.; Tasumi, M.; Trezza, R.; Bastiaanssen, W. G. M. 2002. SEBAL (Surface Energy Balance Algorithms for Land). Advance Training and Users Manual - Idaho Implementation, 1, $97 \mathrm{p}$.

Silva, L. G.; Galvíncio, J. D. 2012. Análise comparativa da variação nos índices NDVI e SAVI no Sítio PELD - 22, em Petrolina - PE, na primeira década do século XXI. Revista Brasileira de Geografia Física, 5, 1446-1456.

Giongo, P. R.; Vettorazzi, C. A. 2014. Albedo da superfície por meio de imagens TM-Landsat 5 e modelo numérico do terreno. Revista Brasileira de Engenharia Agrícola e Ambiental, 18, 833-838.

Marengo, J. A.; Torres, R. R.; Alves, L. M. 2016. Drought in Northeast Brazil-past, present, and future. Theoretical and Applied Climatology, 129, 1189-1200.

Aquino, C.; Valladares, G.; Aquino, R.; Oliveira, J. 2016. Variação temporal do Índice de Vegetação por Diferença Normalizada NDVI no Parque Nacional da Serra da Capivara e seu entorno, Piauí, Brasil. Revista de Geociências do Nordeste - REGNE, 2, 639-649.

Leite, A. P.; Santos, G. R.; Santos, J. É. O. 2017. Análise temporal dos índices de vegetação NDVI e SAVI na Estação Experimental de Itatinga utilizando imagens Landsat 8 . Revista Brasileira de Energias Renováveis, 6 , 606-623.

Macedo, M. J.; Santo, F. A.; Sousa, F. D. 2017. Geoprocessamento aplicado as características físicas e biofísicas da Bacia Hidrográfica do Rio Parnaíba. Revista de Geografia, 34, 170192. 